\title{
Calidad de vida de los adultos mayores en el distrito de Huancas, Chachapoyas, Perú, 2019
}

\section{Quality of life of older adults in the Huancas district, Chachapoyas, Peru, 2019}

\author{
Loidi Sopla Vilca ${ }^{1}$, Julio Mariano Chávez Milla²
}

\section{RESUMEN}

El presente estudio fue de enfoque cuantitativo, de nivel descriptivo, de tipo: observacional, prospectivo, trasversal y de análisis univariado, cuyo objetivo fue: Determinar la calidad de vida de los adultos mayores del distrito de Huancas - Chachapoyas, 2019. La muestra estuvo conformada por 40 Adultos mayores. Los datos fueron recolectados a través del cuestionario de salud SF36. El análisis de los objetivos se realizó con la estadística descriptiva de frecuencias. Los resultados indican que del 100\% (40) adultos mayores el 47.5\% (19) tienen una calidad de vida en promedio, el 22.5\% (9) bajo, el $20 \%$ (8) alto y el 10\% (4) muy bajo. Los adultos mayores en las dimensiones de función física (62.55 y $27.5 \%$ ), rol físico (55\% y 22.5\%) y dolor corporal (57.5\% y $32.5 \%$ ) tienen una calidad de vida de promedio a alto; y mientras que en las dimensiones de salud general, vitalidad, función social, rol emocional, salud mental y transición en la salud tienen una calidad de vida de promedio a bajo. En conclusión, en forma general casi la mitad de los adultos mayores tiene una calidad de vida en promedio seguida de una calidad de vida baja con cierta tendencia a una alta calidad de vida.

Palabras claves: Calidad de vida, adulto mayor

\begin{abstract}
The present study was of quantitative approach, descriptive level, type: observational, prospective, transverse and univariate analysis, whose objective was: To determine the quality of life of the elderly in the District of Huancas Chachapoyas, 2019. The sample was made up of 40 older adults. Data were collected using a scale of quality of life. The objectives were analyzed using the descriptive frequency statistics. The results indicate that of $100 \%(40)$ older adults, $47.5 \%$ (19) have an average quality of life, $22.5 \%$ (9) low, 20\% (8) high and 10\% (4) very low. Older adults in the dimensions of physical function $(62.55 \%$ and $27.5 \%)$, physical role $(55 \%$ and $22.5 \%)$ and body pain $(57.5$ and $32.5 \%)$ have a quality of life from average to high; and while the dimensions of general health, vitality, social function, emotional role, mental health and transition in health have a quality of life from average to low. In conclusion, in general almost half of the elderly have an average quality of life followed by a low quality of life with a certain tendency to a high quality of life.
\end{abstract}

Keywords: Quality of life, elderly

Tesista en Enfermería, Universidad Nacional Toribio Rodríguez de Mendoza de Amazonas. Correo electrónico: loidisopla94@gmail.com ${ }^{2}$ Docente Asociado a Tiempo Completo en la Facultad de Ciencias de la Salud, Universidad. Nacional Toribio Rodríguez de Mendoza de Amazonas.Correo electrónico:julio.chavez@untrm.edu.pe. 


\section{INTRODUCCIÓN}

El proceso de envejecimiento en el mundo es uno de los eventos más trascendentales no sólo desde el punto de vista demográfico sino económico y social. En Sudamérica el 8\% de la población es mayor de 65 años y se estima que en el 2025 este porcentaje alcanzará el 23\%. Existen país, como Japón, cuya población adulta mayor ya está alrededor del $25 \%$. (OPS, 2015).

Debido a las características y cambios asociados al proceso de envejecer, es importante conocer la calidad de vida con la que se vive esta etapa. Este conocimiento es de interés principal en el área de la salud, ya que ésta es la más importante en la percepción de bienestar en los ancianos; sin embargo, la calidad de vida no sólo está enfocada a evaluar este aspecto, sino que también se incluyen los factores sociales, económicos y personales. Por otro lado, no todas las personas viven la vejez de la misma manera, pues su funcionamiento durante ésta se encuentra relacionado con las acciones y omisiones que cada persona realiza durante el transcurso de su vida; es decir, "la vejez se construye desde la juventud". Así, a pesar de que el proceso de envejecimiento es normal, natural e inevitable, puede tener distintos resultados, generalmente reflejo de los cuidados o descuidos tenidos a lo largo de la vida. (Botero, 2016, p.34).

En el Perú 3345552 de los habitantes del país son personas adultas mayores, lo que equivale al 10,4\% de la población total. Según las proyecciones demográficas estimadas, para el año 2020 se espera que la cantidad de adultos peruanos de más de 60 años aumente a 3593054 personas (INEI 2018, p. 21)

Las personas mayores no cuentan con suficientes recursos económicos para satisfacer en forma adecuada sus necesidades básicas; en contraparte las que están institucionalizados, experimentan sentimientos de soledad por encontrarse separados de su núcleo familiar, mostrándose apáticos, poco participativos en las actividades programadas por los centros y tiene mayores factores de riesgo como sedentarismo y aislamiento. Además, evidencian discapacidades más visibles y requieren mayor ayuda para realizar actividades de la vida diaria; en el caso de las personas mayores que vivían con su familia y eran atendidos en el centro diurno aportaron ayuda económica o de intervención en alguna tarea doméstica agrícola entre otras. Estos sujetos se mostraron más activos, participativos y se mantuvieron ocupados en diferentes tareas. (Chuquipul, 2018, p. 14).

A medida que las personas envejecen, aumenta la probabilidad de importantes pérdidas funcionales. Así, el adulto mayor con alguna enfermedad, especialmente si es crónica, teme la pérdida de su buen funcionamiento mental y físico, la presencia de dolores crónicos es penoso, así como las condiciones progresivas de degeneración, haciendo referencia a que el deterioro físico y mental pudiese agudizarse a causa de la depresión e impotencia, y a los temores por la pérdida del control. Las condiciones desfavorables antes mencionadas hacen difícil y complicada la vida de los ancianos, originando en algunos de ellos sentimientos de aislamiento e inutilidad; además, la "fragilidad" física que aumenta en este periodo de la vida hace que las personas de la tercera edad se sientan inútiles. (García, 2015, p. 32)

La calidad de vida es la percepción que un individuo tiene de su lugar en la existencia, en el contexto de la cultura y del sistema de valores en los que vive y en relación con sus objetivos, sus expectativas, sus normas, sus inquietudes. Se trata de un concepto muy amplio que está influido de modo complejo por la salud física del sujeto, su estado psicológico, su nivel de independencia, sus relaciones sociales, así como su relación con los elementos esenciales de su entorno. (OMS, 2007).

La calidad de vida ha sido estudiada desde diferentes disciplinas. Socialmente tiene que ver con una capacidad adquisitiva que permita vivir con las necesidades básicas cubiertas además de disfrutar de una buena salud física - psíquica y de una relación social satisfactoria. El estudio de la calidad de vida en la tercera edad, obliga a incluir aspectos relacionados con esta etapa del ciclo vital, en la cual el sujeto no sólo se enfrenta al envejecimiento cronológico sino también al envejecimiento funcional, dado este último por la disminución de las capacidades físicas, psíquicas y sociales para el desempeño de las actividades de la vida diaria. (Olivares \& Gonzáles, 2015, p. 3)

De una forma u otra, los factores sociales, individuales relacionados con el nivel de vida, son los que determinan la calidad de vida de este grupo poblacional y mejorará en la medida que ellos como un todo funcionen y se integren. La salud y la satisfacción juegan un papel primordial donde se pueda lograr una calidad de vida ajustada a la esperanza de vida. (Lozada, 2015, p. 23).

El departamento de Amazonas cuenta con una población de 422,629 habitantes, donde el 7.8\% representa la población adulta mayor 33,072. (INEI, 2017,p. 3).

Estudios sobre la calidad de vida surgen para conocer y dar mejor atención a las necesidades de la persona en este caso específicamente del adulto mayor, ya que por los cambios físicos, psicológicos y sociales asociados al proceso del envejecimiento constituyen una población particularmente vulnerable que requiere de estrategias de atención que les garanticen una calidad de vida digna. Por ello es necesario conocer como es la calidad de vida de los adultos 
mayores del distrito de Huancas.

Por todo lo descrito anteriormente el objetivo de la presente investigación fue determinar la calidad de vida de los adultos mayores del distrito de Huancas, Chachapoyas, Perú 2019.

\section{MATERIAL Y MÉTODO}

\subsection{Tipo y diseño de investigación.}

La presente investigación fue de enfoque cuantitativo; de nivel descriptivo; de tipo: Según la intervención de la investigadora fue observacional; según la planificación de la toma de datos fue prospectivo; según el número de ocasiones en que se midió la variable de estudio fue transversal y según el número de variables de interés fue de análisis univariado. (Supo, 2016, pp. 2 - 19).

\section{Diseño de la investigación}

Se utilizará el diseño descriptivo, cuyo diagrama será el siguiente:

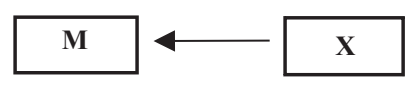

Donde:

$\mathrm{M}=$ La muestra de estudio.

$\mathrm{X}=$ Calidad de vida

\subsection{Población, muestra y muestreo.}

El universo muestral, estuvo constituido por todos los adultos mayores del distrito de Huancas, que sumaron un total de 40 según (INEI, 2017).

\section{Muestreo:}

Se utilizó el muestreo no probabilístico por conveniencia.

\section{Métodos de investigación}

Durante el proceso de investigación, se empleó el método inductivo - deductivo. Porque permitió realizar el análisis de lo particular a lo general.

\section{RESULTADOS.}

Tabla 1. Calidad de vida de los adultos mayores del distrito de Huancas, Chachapoyas, 2019.

\begin{tabular}{ccc}
\hline Calidad de vida & fi & \% \\
\hline Muy bajo & 4 & 10 \\
Bajo & 9 & 22.5 \\
Promedio & 19 & 47.5 \\
Alto & 8 & 20 \\
Muy alto & 0 & 0 \\
\hline Total & 40 & \\
\hline
\end{tabular}

Descripción: Se observa que del 100\% (40) adultos mayores el $47.5 \%$ (19) tienen una calidad de vida en promedio, el $22.5 \%$ (9) bajo, el $20 \%$ (8) alto y el 10\% (4) muy bajo.

Tabla 02: Calidad de vida según sus dimensiones de los adultos mayores del distrito de Huancas, Chachapoyas, 2019.

\begin{tabular}{|c|c|c|c|c|c|c|c|c|c|c|c|c|}
\hline \multirow{3}{*}{ Dimensiones } & \multicolumn{10}{|c|}{ Calidad de vida } & \multirow{2}{*}{\multicolumn{2}{|c|}{ Total }} \\
\hline & \multicolumn{2}{|c|}{ Muy Bajo } & \multicolumn{2}{|c|}{ Bajo } & \multicolumn{2}{|c|}{ Promedio } & \multicolumn{2}{|c|}{ Alto } & \multicolumn{2}{|c|}{ Muy alto } & & \\
\hline & fi & $\%$ & fi & $\%$ & fi & $\%$ & fi & $\%$ & fi & $\%$ & fi & $\%$ \\
\hline Función física & 0 & 0 & 4 & 10 & 25 & 62.5 & 11 & 27.5 & 0 & 0 & 40 & 100 \\
\hline Rol físico & 0 & 0 & 6 & 15 & 22 & 55 & 9 & 22.5 & 3 & 7.5 & 40 & 100 \\
\hline Dolor corporal & 0 & 0 & 1 & 2.5 & 23 & 57.5 & 13 & 32.5 & 3 & 7.5 & 40 & 100 \\
\hline Salud general & 0 & 0 & 10 & 25 & 24 & 60 & 6 & 15 & 0 & 0 & 40 & 100 \\
\hline Vitalidad & 4 & 10 & 10 & 25 & 19 & 47.5 & 7 & 17.5 & 0 & 0 & 40 & 100 \\
\hline Funcion social & 1 & 2.5 & 13 & 32.5 & 19 & 47.5 & 7 & 17.5 & 0 & 0 & 40 & 100 \\
\hline Rol emocional & 2 & 5 & 10 & 25 & 23 & 57.5 & 5 & 12.5 & 0 & 0 & 40 & 100 \\
\hline Salud mental & 1 & 2.5 & 6 & 15 & 28 & 70 & 5 & 12.5 & 0 & 0 & 40 & 100 \\
\hline \multirow[t]{2}{*}{ Transición de la salud } & 1 & 2.5 & 8 & 20 & 27 & 67.5 & 4 & 10 & 0 & 0 & 40 & 100 \\
\hline & 9 & 22.5 & 68 & 170 & 210 & 525 & 67 & 167.5 & 6 & 15 & 40 & 100 \\
\hline
\end{tabular}

\section{Descripción:}

Se observa que del 100\% (40) de adultos mayores en cuanto a la calidad de vida según dimensiones se observa que de la dimensión de la función física el 62.5\% (25) tienen una calidad de vida en promedio, el 27.5\% (11) 
alto, el $10 \%$ (4) bajo.

En la dimensión del rol físico el 55\% (22) tienen una calidad de vida promedio, $22.5 \%$ (9) alto, el 15\% (6) bajo y el7.5\% (3) muy alto.

En la dimensión de dolor corporal el 57.5\% (23) tiene una calidad de vida promedio, 32.5\% (13) alto, 7.5\% (3) muy alto y el $2.5 \%$ (1) bajo.

En la dimensión de salud general el 60\% (24) tiene una calidad de vida promedio, $25 \%$ (10) bajo y el $15 \%$ (6) alto.

En la dimensión de vitalidad el 47.5\% (19) tiene una calidad de vida promedio, $25 \%$ (10) bajo, $17.5 \%$ (7) alto y el 10\% (4) muy bajo.

En la dimensión de función social el 47.5\% (19) tiene una calidad de vida promedio, 32.5\% (13) bajo, $17.5 \%$ (7) alto y el $2.5 \%$ (1) muy bajo.

En la dimensión del rol emocional el 57.5\% (23) tiene una calidad de vida promedio, 25\% (10) bajo, $12.5 \%$ (5) y el $5 \%$ (2) muy bajo.

En la dimensión de salud mental el 70\% (28) tiene una calidad de vida promedio, $15 \%$ (6) bajo, $12.5 \%$ (5) alto y el 2.5\% (1) muy bajo.

En la dimensión de transición de la salud el 67.5\% (27) tiene una calidad de vida promedio, $20 \%$ (8) bajo, $10 \%$ (4) alto y el $2.5 \%$ (1) muy bajo.

\section{DISCUSIÓN}

La presente tesis muestra que los adultos mayores del distrito de Huancas, Chachapoyas, 2019 el 47.5\% tienen una calidad de vida en promedio, el $22.5 \%$ bajo, el $20 \%$ alto y el 10\% muy bajo (Tabla 1 ). Quiere decir que los adultos mayores tienen una función física, un rol físico en promedio, tienen un dolor corporal soportable, una salud general, una vitalidad normal, tienen una función social sin problemas con una vida social normal, con un rol emocional, una salud mental y una transición social en promedio.

Desde el punto de vista de la calidad de vida, los adultos mayores estudiados (Tabla 2), percibieron con mayor frecuencia que su calidad de vida era promedio y comparativamente en el tiempo, cerca de la mitad consideraba que se encontraba algo o mucho peor que hace un año. Desde el punto de vista funcional, aproximadamente dos tercios de la población evaluada o no tenían o tenían poca limitación para llevar bolsas de compra, para subir un piso por la escalera, para agacharse, arrodillarse o ponerse de cuclillas o caminar medio kilómetro. Sin embargo, la tercera parte reconoció presentar mucha limitación para actividades moderadas e intensas y cuatro de cada diez consideró presentar mucha limitación para la realización de actividades cotidianas como bañarse o vestirse.

Se tiene que determinar que la calidad de vida es la percepción que un individuo tiene de su lugar en la existencia, en el contexto de la cultura y del sistema de valores en los que vive y en relación con sus objetivos, sus expectativas, sus normas, sus inquietudes. La calidad de vida está influenciado de modo complejo por la salud física del adulto mayor, su estado psicológico, su nivel de independencia, sus relaciones sociales, así como su relación con los elementos esenciales de su entorno.

Así mismo existe diferencias de los resultados del presente estudio con las de Mazacón, B. (2017), Ecuador, que estudió la calidad de vida en los adultos mayores del Cantón Ventanas intervenidos y no intervenidos con el modelo de atención integral en salud. Los resultados indicaron que la mayor puntuación promedio en el cuestionario SF36 la obtuvieron las dimensiones de calidad de vida de desempeño emocional $(76,0)$, desempeño físico $(64,3)$ y salud general $(58,5)$; mientras que, los puntajes más bajos lo obtuvieron en las dimensiones salud mental $(38,8)$, función social $(46,5)$, función física $(48,5)$, vitalidad $(49,0)$ y dolor físico $(49,6)$, no se observó diferencia estadísticamente relevante entre los intervenidos y los no intervenidos por el modelo de atención integral de salud.

También es diferente con los resultados de Eras, L. (2015). Piura. Su objetivo fue determinar la relación entre el clima social familiar y calidad de vida de los participantes del programa del adulto mayor del centro de salud Los Algarrobos. Se evidenció que, de los 100 participantes evaluados, el 37\% presentaron un nivel medio de clima social familiar, en la cual el $19 \%$ presentaron un mal clima social familiar, seguido del $18 \%$ que presentó un muy buen clima social familiar, luego el $15 \%$ presentó un buen clima social familiar y finalmente el $11 \%$ presentó un muy mal clima social familiar, lo cual quiere decir que predomina un clima promedio. Asimismo, se observó que la calidad de vida de los 100 participantes evaluados, el 54\% presentó un nivel regular de calidad de vida, y el $25 \%$ presentó nivel bajo de calidad de vida, seguido del $21 \%$ que presentó un nivel alto de calidad de vida.

Asimismo, existe una diferencia entre los resultados de Valdez, J. (2017), quien determinó los factores psicológicos y sociales relacionados a la calidad de vida del adulto mayor. Encontró que el 55\% de la población adulta mayor se encuentra con una calidad de vida mala y el $45 \%$ se encuentra con una calidad de vida regular ausentándose una buena calidad de vida; el factor psicológico se encuentra en nivel bajo de autoestima el $45 \%$, en nivel medio de autoestima el $43 \%$ y el nivel elevado de autoestima 12\%. En concñusión del total de la población estudiada existe un notorio porcentaje del $55 \%$ que presenta una mala calidad de vida seguido de un $45 \%$ regular calidad de 
vida. Por lo tanto, existe una diferencia con la investigación de Lozada, J. (2015). Trujillo. Donde su objetivo fue: determinar efecto del programa de estilo de vida para un envejecimiento activo en la calidad de vida del adulto mayor en los aspectos físicos, sociales y psicológicos. Los resultados indicaron que los adultos mayores en $48 \%$ tuvieron una calidad de vida deteriorada, $32 \%$ una calidad de vida poco deteriorada, y el $20 \%$ una calidad de vida normal. En conclusión, en esta población es muy predominante la calidad de vida deteriorada.

Hay una diferencia con el estudio de Chuquipul, P. (2018). Lima. Realizó el estudio cuyo objetivo fue determinar la relación entre el deterioro cognitivo y la calidad de vida del adulto mayor de la asociación organización social del adulto mayor, Villa los Reyes, Ventanilla 2018. Se observó que los adultos mayores tienen deterioro cognitivo moderado con un $70 \%$; en cuanto a sus dimensiones como orientación en el tiempo tuvo un $84 \%$, orientación en el espacio $58 \%$, lenguaje/nominación $46 \%$ y atención/calculo $46 \%$; por otro lado la calidad de vida se evidenció que la categoría que prevaleció es la promedio con un $88 \%$, en relación a sus dimensiones la función física presentó un $74 \%$, dimensión dolor corporal $80 \%$, dimensión salud general $60 \%$, dimensión vitalidad $86 \%$, dimensión función social $70 \%$, dimensión a salud mental $\quad 78 \%$, dimensión transición de la salud $70 \%$ dando así un resultado de una calidad de vida alta. El estudio concluyó que no existe relación entre el deterioro cognitivo y la calidad de vida, en su mayoría de los adultos mayores tiene deterioro cognitivo moderado y calidad de vida promedio.

Es fundamental indicar que todos los seres humanos optan por tener una buena calidad de vida esta contribuye a la mejora el nivel de vida de los demás ya sea la edad que tenga esta. El tener bajo nivel de calidad de vida influye posibles discriminación social al no querer aceptar a la persona por su condiciones económicas de cómo viven o la zona por donde radican o tal vez por la mera de vestir, estos casos de aislamiento social es perjudicial para cualquier persona y más para una persona de la tercera edad quien está propenso a las ofensas y la escaza seguridad de parte de su familia y la sociedad en la que vive, observando el trato tanto de la familia como de la sociedad los adultos mayores buscan ser aceptados por todas las personas y deciden perteneces a instituciones que las ayuda a sentirse queridos y sentir que son aceptados si discriminación alguna.

A medida que las personas envejecen, aumenta la probabilidad de importantes pérdidas funcionales. Así, el adulto mayor con alguna enfermedad, especialmente si es crónica, teme la pérdida de su buen funcionamiento mental y físico, la presencia de dolores crónicos y penosos, así como las condiciones progresivas de degeneración, haciendo referencia a que el deterioro físico y mental pudiese agudizarse a causa de la depresión e impotencia, y a los temores por la pérdida del control.

\section{CONCLUSIONES}

1. En forma general casi la mitad de los adultos mayores tiene una calidad de vida en promedio seguida de una calidad de vida baja con cierta tendencia a una alta calidad de vida.

2. Así mismo en todas las dimensiones la calidad de vida de los adultos mayores es en promedio. Aunque en las dimensiones de función física, rol físico y dolor corporal la calidad de vida es de promedio a alto; mientras que en las dimensiones de salud mental, vitalidad, función social, rol emocional, salud mental y transición en salud la calidad de vida de los adultos mayores es de promedio a bajo.

3. De acuerdo a los grupos etarios, los adultos mayores de 60 a 69 años la calidad de vida es de promedio a alto; mientras que en los de 70 a 79 años la calidad de vida es de promedio a bajo, y en los adultos de 80 a más años tienen una calidad de vida de muy bajo a bajo.

4. Los adultos mayores de sexo masculino tienen una calidad de vida de promedio a alto, con tendencia baja; mientras que los de sexo femenino de promedio a bajo, en algunos casos a muy bajo y en otros alto.

5. Los adultos mayores casados tienen una calidad de vida de promedio a bajo; mientras que el total de los solteros tiene una calidad de vida en promedio; los viudos de promedio a alto.

\section{REFERENCIAS BIBLIOGRAFICAS}

Botero, B. (2016). Calidad de vida relacionada con la salud en adultos mayores de 60 años: una aproximación teórica. Hacia la promoción de la salud [Internet]. [acceso 17 de Agosto 20019 ]: 12 . D is p o n ib le e n : http://www.scielo.org.co/scielo.php?script= s c i_a r t t ex t \& p i d = S 0121 75772007000100002 .

Chuquipul, P. (2818). Perú. Deterioro cognitivo y calidad de vida del adulto mayor de la asociación organización social del adulto mayor, Villa Los Reyes, Ventanilla 2018. Tesis de licenciatura.

Eras, L. (2015). Relación entre el clima social familiar y calidad de vida de los participantes en el programa del Adulto Mayor del Centro de Salud los Algarrobos. Piura, 2014. [Tesis licenciatura]. ULADECH. Piura. Perú.

García, M. (2015). Rendimiento cognitivo y calidad 
de vida de adultos mayores asistentes a grupos de tercera edad. Acta Neurol Colomb. [Internet] 2015 [acceso $20 \mathrm{de}$ Agosto de 2019] 31(4): 398-403. Disponible e $\mathrm{n}$ http://www.scielo.org.co/pdf/anco/v31n4/v3 1n4a07.pdf

Instituto Nacional de Estadística e Informática. (2015). Esperanza de vida de población peruana aumentó en 15 años en las últimas cuatro décadas [sede web]. Lima-Perú; 2015 [acceso 5 de Agosto de 2019]. Disponible en: https://www.inei.gob.pe/prensa/noticias/esp eranza-de-vida-de-poblacion-eruanaaumento-en-15-anos-en-las-ultimas-cuatrodecadas-8723.

Lozada, J. (2015). Calidad de vida del adulto mayor: Efectos del programa estilo de vida para un envejecimiento activo - EsSalud, Distrito la Esperanza, provincia de Trujillo - 2014. (Tesis de grado de Maestría).

Mazacón, B. (2017). Calidad de vida del adulto mayor en una comunidad del cantón Ventanas - Los Ríos intervenida con el modelo de atención integral de salud. [Tesis de licenciatura]. UNMSM. LimaPerú.

Supo, J. (2016). Metodología de la investigación científica. 3ra Edic. Edit. Talleres gráficos Arequipa Perú.

Organización Mundial de la Salud. (2015). Informe mundial sobre el envejecimiento y la salud [sede web]. Ginebra -Suiza: Organización Mundial de la Salud; [acceso 5 de Agosto de 2019]. Disponible en: http://apps.who.int/iris/bitstream/10665/186 466/1/9789240694873_spa.pdf

Valdez, J. (2017). Factores Psicológicos y Sociales Relacionados a la calidad de vida del Adulto Mayor, Centro de Salud Cono Sur I-4, San Román 2017.[Tesis de licenciatura]. Puno Perú: Universidad Nacional del Altiplano.

Organización Mundial de la Salud. (2007). Repercusión mundial del envejecimiento en la Salud. [documento en línea]. Disponible $\mathrm{e}$

$\mathrm{n}$

htt://www.who.int/features/qa/42/es/index.h tml. 